\title{
Curvature and the Evolution of Fronts
}

\author{
J. A. Sethian* \\ Courant Institute of Mathematical Sciences, New York University, New York, NY 10012, USA
}

\begin{abstract}
The evolution of a front propagating along its normal vector field with speed $F$ dependent on curvature $K$ is considered. The change in total variation of the propagating front is shown to depend only on $d F / d K$ only where $K$ changes sign. Analysis of the case $F(K)=1-\varepsilon K$, where $\varepsilon$ is a constant, shows that curvature plays a role similar to that of viscosity in Burgers equation. For $\varepsilon=0$ and non-convex initial data, the curvature blows up, corners develop, and an entropy condition can be formulated to provide an explicit construction for a weak solution beyond the singularity. We then numerically show that the solution as $\varepsilon$ goes to zero converges to the constructed weak solution. Numerical methods based on finite difference schemes for marker particles along the front are shown to be unstable in regions where the curvature builds. As a remedy, we show that front tracking based on volume of fluid techniques can be used together with the entropy condition to provide transition from the classical to weak solution.
\end{abstract}

\section{Introduction}

We study the evolution of a front propagating along its normal vector field with speed a function of curvature. We first prove a general result relating the growth/decay of the total variation to the speed. We then study the case of a front moving with speed $1-\varepsilon K$, where $\varepsilon$ is a constant and $K$ is the curvature, and show that the curvature term plays a smoothing role in the solution similar to that of viscosity in Burgers equation. For $\varepsilon=0$, in which case the front moves at constant speed, the curvature blows up, differentiability is lost, and an entropy condition can be formulated to provide an explicit construction of a weak solution beyond the singularity. For $\varepsilon>0$, we then solve the equations of motion numerically and show that in the limit as $\varepsilon$ goes to zero, the solution converges to our constructed weak solution. We show that corners which develop in the propagating front

* National Science Foundation Mathematical Sciences Post-Doctoral Fellow 
swallow variation in the solution, providing a discontinuous stabilizing mechanism.

We discuss the difficulties in numerically solving such problems. We show that finite difference schemes for marker particles placed along the front can go unstable as the curvature builds, unless artificial smoothing and small time steps are employed. In contrast, we show that front tracking based on volume of fluid techniques can be used together with the entropy condition to allow transition from the classical solution to the weak solution.

Our motivation comes from crystal growth and flame propagation. In the former, a typical problem is the stability of the boundary of a solid growing in a supercooled liquid, see $[9,10,16]$. At the boundary, the Gibbs-Thomson thermodynamic boundary condition, see [20], states that $T(\bar{x})=T_{M}(1-\varepsilon K(\bar{x}))$, where $T(\bar{x})$ is the temperature at a point $\bar{x}$ on the boundary, $T_{M}$ is the melting temperature, $\varepsilon$ is a constant and $K(\bar{x})$ is the curvature. Thus, the solidification rate at the boundary is a function of curvature. Discussion of the theory of interface stability may be found in Pamphlin [16]; other examples may be found in $[9,10$, $13,14]$. Similarly, in flame theory, one model idealizes the front as an infinitely thin boundary separating regions of constant steady-state velocity, density and temperature; by ignoring all but hydrodynamic effects, Landau [8] showed that flames are unstable to perturbations. Markstein [11] postulated from physical arguments that the flame speed must depend on the curvature so that $V(\bar{x})$ $=V^{0}(1-\varepsilon K(\bar{x}))$, where $V^{0}$ is the constant speed of a flat flame, and thus demonstrated the stabilizing effect of curvature. A comprehensive though now outdated account may be found in Markstein [12], finally, we also mention the work of Sivashinsky [19], Frankel and Sivashinsky [4], and Zeldovich [21].

A limitation of linear stability analysis is the assumption of a smooth solution, excluding the possibility of discontinuities in the solution as a stabilizing or destabilizing effect. Here, we take a geometrical approach to the idealized problem of the motion of a closed curve along its normal vector field in order to study stability/instability, breakdown of solution and long-time steady states.

\section{General Results}

\section{A. Equations of Motion}

Starting with a simple, smooth, closed initial curve $\gamma(0)$ in $R^{2}$, let $\gamma(t)$ be the oneparameter family of curves, where $t \in[0, \infty)$ is time, generated by moving the initial curve along its normal vector field with speed $F$ a given function of the curvature. Let $\mathbf{x}(s, t)$ be the position vector which parameterizes $\gamma(t)$ by $s, 0 \leqq s \leqq S, \mathbf{x}(0, t)$ $=\mathbf{x}(S, t)$. The curve is parameterized so that the interior is on the left in the direction of increasing $s$. With $K(s, t)$ as the curvature at $\mathbf{x}(s, t)$, the equations of motion are

$$
\begin{gathered}
\mathbf{n}(s, t) \cdot \frac{\partial \mathbf{x}(s, t)}{\partial t}=F(K(s, t)), \\
\mathbf{x}(s, 0)=\gamma(0) \quad \text { prescribed; } \quad s \in[0, S], \quad t \in[0, \infty),
\end{gathered}
$$


where $\mathbf{n}(s, t)$ is the unit normal vector at $\mathbf{x}(s, t)$. Written in terms of the coordinates $\mathbf{x}(s, t)=(x(s, t), y(s, t))$, an equivalent formulation is

$$
\begin{gathered}
x_{t}=F\left(\frac{y_{s s} x_{s}-x_{s s} y_{s}}{\left(x_{s}^{2}+y_{s}^{2}\right)^{3 / 2}}\right) \frac{y_{s}}{\left(x_{s}^{2}+y_{s}^{2}\right)^{1 / 2}}, \\
y_{t}=-F\left(\frac{y_{s s} x_{s}-x_{s s} y_{s}}{\left(x_{s}^{2}+y_{s}^{2}\right)^{3 / 2}}\right) \frac{x_{s}}{\left(x_{s}^{2}+y_{s}^{2}\right)^{1 / 2}}, \\
(x(s, 0), y(s, 0))=\gamma(0) \quad 0 \leqq s \leqq S .
\end{gathered}
$$

If $\alpha(s)$ corresponds to arclength, then $d \alpha=g(s, t) d s$, where $g(s, t)=\left(x_{s}^{2}+y_{s}^{2}\right)^{1 / 2}$. Using the relations

$$
\begin{gathered}
x_{t} x_{t s}+y_{t} y_{t s}=\frac{F(K) K}{x_{s}^{2}+y_{s}^{2}} \\
x_{s} y_{t s}-y_{s} x_{t s}=-\frac{\partial F(K)}{\partial s}\left(x_{s}^{2}+y_{s}^{2}\right)^{1 / 2}
\end{gathered}
$$

one can produce an evolution equation for the metric $g$, namely

$$
g_{t}(s, t)=g(s, t) K(s, t) F(K(s, t)),
$$

and an evolution equation for the curvature $K$, namely

$$
K_{t}(s, t)=-\left[F_{s}(K(s, t)) g^{-1}(s, t)\right]_{s} g^{-1}(s, t)-K^{2}(s, t) F(K(s, t)) .
$$

Brakke [1] has studied the case $F(K)=K$, which occurs in the modeling of grain boundaries in metals. There, the sign is chosen so that the surface moves inward when the mean curvature is positive. It has recently been shown [7] that a convex surface remains smooth as it collapses to a single point; to the best of our knowledge, the question of smoothness of the moving surface for a non-convex initial surface remains open.

\section{B. Decay of Total Variation - Smoothing of Solution}

Let $\operatorname{Var}(t)$ be the total variation of the front at time $t$, defined as

$$
\operatorname{Var}(t)=\int_{0}^{S}|K(s, t)| g(s, t) d s .
$$

Proposition 1. Consider a front moving with speed $F(K)$, as in Eq. (2.1). Assume that $\gamma(0)$ is non-convex, so that $K(s, 0)$ changes sign, and assume $K$ is zero at a finite number of points. Assume that $F$ is twice differentiable, and that $K(s, t)$ is twice differentiable for $0 \leqq s \leqq S$ and $0 \leqq t \leqq T$. Then, for $0 \leqq t \leqq T$

1) if $F_{K}(0) \leqq 0\left(F_{K}(0) \geqq 0\right)$, then

$$
\frac{d \operatorname{Var}(t)}{d t} \leqq 0\left(\frac{d \operatorname{Var}(t)}{d t} \geqq 0\right),
$$

2) if $F_{K}(0)<0\left(F_{K}(0)>0\right)$ and $K_{s}(0) \neq 0$, then

$$
\frac{d \operatorname{Var}(t)}{d t}<0\left(\frac{d \operatorname{Var}(t)}{d t}>0\right)
$$


Remarks. Proposition 1 states that if $F_{K}<0$ wherever $K=0$, then the front "flattens out." If $\gamma(t)$ is convex, the proposition is trivial, since $\operatorname{Var}(t)=\int_{0}^{S} K g d s=2 \pi$. Note that we have assumed that the curvature is twice differentiable in the interval $0 \leqq t$ $\leqq T$; in Sect. III we discuss what happens if the front ceases to be smooth and develops a corner.

Proof of Proposition 1. From Eqs. (2.6) and (2.7), we have

$$
\begin{gathered}
g_{t}=g K F, \\
K_{t}=-g^{-1}\left[g^{-1} F_{s}\right]_{s}-K^{2} F .
\end{gathered}
$$

Suppose that at $s_{1}(t), \ldots, s_{i}(t), \ldots, s_{n}(t), K\left(s_{i}(t), t\right)=0$ and $K$ changes sign going from $s<s_{i}(t)$ to $s>s_{i}(t)$. Assume $K>0$ in $\left(s_{1}(t), s_{2}(t)\right),\left(s_{3}(t), s_{4}(t)\right), \ldots,\left(s_{n-1}(t)\right.$, $\left.s_{n}(t)\right)$ and $K<0$ in $\left(s_{2}(t), s_{3}(t)\right),\left(s_{4}(t), s_{5}(t)\right), \ldots,\left(s_{n}(t), s_{1}(t)+S=s_{1}(t)\right)$. Then

$$
\begin{aligned}
\operatorname{Var}(t)= & \int_{0}^{S}|K| g d s=\int_{s_{1}(t)}^{s_{2}(t)} K g d s+\int_{s_{3}(t)}^{s_{4}(t)} K g d s+\ldots+\int_{s_{n-1}(t)}^{s_{n}(t)} K g d s \\
& -\int_{s_{2}(t)}^{s_{3}(t)} K g d s-\int_{s_{4}(t)}^{s_{3}(t)} K g d s-\ldots-\int_{s_{n}(t)}^{s_{1}(t)+s} K g d s .
\end{aligned}
$$

For simplicity, we shall assume $K$ changes sign only at two points, $s_{1}(t)$ and $s_{2}(t)$, thus

$$
\operatorname{Var}(t)=\int_{s_{1}(t)}^{s_{2}(t)} K g d s-\int_{s_{2}(t)}^{s_{1}(t)} K g d s
$$

Then

$$
\begin{aligned}
\frac{d \operatorname{Var}(t)}{d t}= & \int_{s_{1}(t)}^{s_{2}(t)}(K g)_{t} d s-\int_{s_{2}(t)}^{s_{1}(t)+S}(K g)_{t} d s+K\left(s_{2}(t), t\right) g\left(s_{2}(t), t\right) s_{2}^{\prime}(t) \\
& \left.-K\left(s_{1}(t), t\right) g\left(s_{1}(t), t\right) s_{1}^{\prime}(t)\right) \\
& -K\left(s_{1}(t)+S, t\right) g\left(s_{1}(t)+S, t\right)\left(s_{1}(t)+S\right)^{\prime}+K\left(s_{2}(t), t\right) g\left(s_{2}(t), t\right) s_{2}^{\prime}(t),
\end{aligned}
$$

where both the subscript $t$ and the prime refer to differentiation with respect to $t$. By assumption, $K\left(s_{1}(t), t\right)=K\left(s_{2}(t), t\right)=K\left(s_{1}(t)+S, t\right)=0$, thus

$$
\frac{d \operatorname{Var}(t)}{d t}=\int_{s_{1}(t)}^{s_{2}(t)}\left(K_{t} g+K g_{t}\right) d s-\int_{s_{2}(t)}^{s_{1}(t)+s}\left(K_{t} g+K g_{t}\right) d s,
$$

Using Eqs. (2.9) and (2.10), we have

$$
\begin{aligned}
\frac{d \operatorname{Var}(t)}{d t}= & \int_{s_{1}(t)}^{s_{2}(t)}\left(-\left(g^{-1} F_{s}\right)_{s}-K^{2} F g+g K^{2} F\right) d s-\int_{s_{2}(t)}^{s_{1}(t)+S}\left(-\left(g^{-1} F_{s}\right)_{s}\right. \\
& \left.-K^{2} F g+g K^{2} F\right) d s \\
= & -\int_{s_{1}(t)}^{s_{2}(t)}\left(g^{-1} F_{s}\right)_{s} d s+\int_{s_{2}(t)}^{s_{1}(t)+s}\left(g^{-1} F_{s}\right)_{s} d s \\
= & -\left(\left.g^{-1} F_{s}\right|_{s_{2}(t)}-\left.g^{-1} F_{s}\right|_{s_{1}(t)}\right)+\left(\left.g^{-1} F_{s}\right|_{s_{1}(t)+s}-\left.g^{-1} F_{s}\right|_{s_{2}(t)}\right) \\
= & -\left.2\left(g^{-1} F_{K} K_{s}\right)\right|_{s_{2}(t)}+\left.2\left(g^{-1} F_{K} K_{s}\right)\right|_{s_{1}(t)} .
\end{aligned}
$$


By assumption, $K>0$ for $s_{1}(t)<s<s_{2}(t)$, hence $\left.K_{s}\right|_{s_{1}(t)} \geqq 0$ and $\left.K_{s}\right|_{s_{2}(t)} \leqq 0$. Assume $F_{K}(0) \geqq 0$. Then, since $g^{-1}>0$, both terms of the right-hand side of Eq. (2.11) are non-negative and $\frac{d \operatorname{Var}(t)}{d t} \geqq 0$. Conversely, if $F_{K}(0) \leqq 0$, then both terms are non-positive and $\frac{d \operatorname{Var}(t)}{d t} \leqq 0$. If $F_{K}$ is strictly less or greater than zero and $K_{s} \neq 0$ at $s_{1}(t)$ and $s_{2}(t)$, then the inequalities are also strict. This completes the proof.

The corollary below applies to a front moving at constant speed:

Corollary. If $F_{K}=0$, then the total variation is constant.

We now examine in detail the case $F(K)=1-\varepsilon K$, where $\varepsilon$ is a constant. The curvature evolution equation is

$$
K_{t}=\varepsilon K_{\alpha \alpha}+\varepsilon K^{3}-K^{2}
$$

where here we have changed variables and taken the derivative of curvature with respect to arclength to eliminate the metric $g$. Equation (2.12) is a reactiondiffusion equation, in which the reaction term $\left(\varepsilon K^{3}-K^{2}\right)$ is mitigated by the diffusion term $\left(\varepsilon K_{\alpha \alpha}\right)$. Indeed, with $\varepsilon=0$, we have $K_{t}=-K^{2}$ with solution

$$
K(s, t)=\frac{K(s, 0)}{1+K(s, 0) t},
$$

which is singular at finite $t$ if the initial curvature is anywhere negative. This singularity corresponds to the development of a corner in the front. As a suggestive example, with $\varepsilon=0$ and initial data $\gamma(0)=\left(s, s^{2}\right),-\infty<s<\infty$, the solution is

$$
x(s, t)=\frac{2 s}{\left(4 s^{2}+1\right)^{1 / 2}} t+s, \quad y(s, t)=\frac{1}{\left(4 s^{2}+1\right)^{1 / 2}} t+s^{2} .
$$

At $t=1 / 2$, a section of the propagating front collapses to a point, where a corner develops. At later times, the front crosses itself.

The above situation is analogous to the development of shocks in hyperbolic conservation laws. Consider Burgers equation with viscosity, namely

$$
u_{t}+u u_{x}=\varepsilon u_{x x}
$$

It is well-known, see [6], that for $\varepsilon=0$, shock discontinuities can develop in the solution, even for smooth initial data. A typical example is initial data

$$
u(x, 0)=\left\{\begin{array}{cc}
1 & x<0 \\
1-x & 0 \leqq x \leqq 1 \\
0 & 1 \leqq x
\end{array}\right\} .
$$

For $\varepsilon>0$ the viscosity term on the right-hand side of Eq. (2.14) diffuses steepening fronts, and the solution remains smooth. However, with $\varepsilon=0$, the characteristics are straight lines (in the $x-t$ plane) along which the solution $u$ is constant. Although the initial data is continuous, at $t=1$ characteristics collide and a shock develops. An entropy condition is used to select the proper way of continuing the 
solution past when a shock develops, resulting in a globally defined weak solution which is the limiting solution of Eq. (2.14) as $\varepsilon \rightarrow 0$. In our problem for a front moving with speed $F(K)=1-\varepsilon K, \operatorname{Var}(t)$ decreases for $\varepsilon>0$ and is constant for $\varepsilon=0$ for non-convex, smooth initial data. While it is reasonable to expect that the front remains smooth for $\varepsilon>0$, for $\varepsilon=0$ a singularity develops in the curvature. In the next section, we continue this analogy and study in detail the propagating front for the case $\varepsilon=0$.

\section{Limiting Case - The Formation of Cusps}

\section{A. Breakdown}

We study the limiting case $\varepsilon=0$ in which case the front moves at a constant speed. The equations of motion are

$$
\begin{gathered}
x_{t}=\frac{y_{s}}{\left(x_{s}^{2}+y_{s}^{2}\right)^{1 / 2}}, \quad y_{t}=-\frac{x_{s}}{\left(x_{s}^{2}+y_{s}^{2}\right)^{1 / 2}}, \\
(x(s, 0), y(s, 0))=\gamma(0) ; \quad 0 \leqq s \leqq S .
\end{gathered}
$$

In vector notation, this is the Eikonal equation. We always assume that the initial curve $\gamma(0)$ is $C^{2}$. The solution to (3.1) and (3.2) is

$$
x(s, t)=\frac{\beta_{s}}{\left(\alpha_{s}^{2}+\beta_{s}^{2}\right)^{1 / 2}} t+\alpha(s), \quad y(s, t)=-\frac{\alpha_{s}}{\left(\alpha_{s}^{2}+\beta_{s}^{2}\right)^{1 / 2}} t+\beta(s),
$$

where $(\alpha(s), \beta(s))=(x(s, 0), y(s, 0))=\gamma(0)$. This solution parameterizes by $t$ the straight lines normal to the initial curve at $s$. These are the geometric rays of optics theory, see [5].

If the initial curve is convex, the solution to the propagation equations (3.1) and (3.2) can be obtained in two ways; either by using the exact solution (3.3) or by relying on a Huygens principle construction, which says that the solution at time $t$ corresponds to the envelope generated by the set of all disks of radius $t$ centered on the initial curve, see [5]. These two constructions produce the same front, since given a point outside a convex initial curve, there is a unique normal to the curve passing through that point. As normals cannot intersect, no corners can develop and the propagating front remains smooth. The front is also reversible; if we know the position of the front at time $t$, we may solve the evolution equations backwards in time (or reverse the geometric construction) to reconstructed the initial curve.

\section{B. The Entropy Condition}

Suppose the initial curve is non-convex. The normals must collide and the curvature becomes singular. Beyond this time, the solution (3.3) differs from the Huygen's principle construction. We now show that an "entropy condition" can be enforced on the propagating normals beyond the curvature singularity to allow a weak solution of (3.3) which is identical to the Huygen's construction. For this discussion, we imagine the front as a flame separating a burnt interior from an unburnt exterior; each point is transformed from unburnt to burnt when touched by the propagating front. The normals will be called ignition curves. 
Let $\phi(x, y, t)$ be the indicator function of the burning region; $\phi(x, y, t)=1$ if the particle at $(x, y)$ is burnt at time $t$ and zero otherwise. Our entropy condition requires that if $\phi\left(x, y, t^{*}\right)=1$, then $\phi(x, y, t)=1$ for $t>t^{*}$, once a particle burns, it remains burnt. The boundary of the set where $\phi=1$ is the position of the front and is identical to the Huygen's principle construction.

Consider now the following construction: Starting with the initial curve, move the front along the ignition curves until there is a collision. Eliminate the curves that collide, and continue moving the front along the remaining curves. As time progresses, the front will be parameterized by a shrinking subset of the original parameterization $[0, S]$, and any point on the solution can be traced back to the initial curve. Conversely, there will be points along the initial curve that do not affect the solution beyond some time, and thus the solution becomes irreversible. This is analogous to shocks, in which characteristics always trace back to the initial line. Furthermore, $\frac{d \operatorname{Var}(t)}{d t} \leqq 0$, since $\operatorname{Var}(t)$ is the integral of a positive quantity over a set whose length is a non-increasing function of time (here, one evaluates $\operatorname{Var}(t)$ over open intervals where the curvature is defined together with jumps in the tangent at the corners).

We now show that the solution obtained by this construction makes use of the entropy condition to produce the boundary of $\phi=1$. We show that, under the entropy condition, colliding ignition curves do not affect the propagating front beyond their collision point, and can thus be eliminated. Let $\bar{x}(s, t)=(x(s, t), y(s, t))$ as in (3.3). The following proposition shows that if two ignition curves collide, they arrive simultaneously at the intersection point, and neither one has any effect beyond that time.

Proposition 2. 1) Suppose $\bar{x}\left(s_{1}, t_{1}\right)=\bar{x}\left(s_{2}, t_{2}\right)$ with $s_{1} \neq s_{2}$ and $t_{1}<t_{2}$. Then for any $t>t_{2}$, there exists $s_{3}$ and $t_{3}$ such that $\bar{x}\left(s_{3}, t_{3}\right)=\bar{x}\left(s_{2}, t\right)$ with $t_{3}<t$.

2) Let $\bar{x}_{s_{1}}\left(\bar{x}_{s_{2}}\right)$ be the ignition curve leaving $\bar{x}\left(s_{1}, 0\right)\left(\bar{x}\left(s_{2}, 0\right)\right)$. Suppose $\bar{x}_{s_{1}}$ is eliminated by $\bar{x}_{s_{2}}$, that is, $\bar{x}\left(s_{1}, t_{1}\right)=\bar{x}\left(s_{2}, t_{2}\right)$ and for all $t>t_{1}$, there is no $s^{*}, t^{*}$ such that $\bar{x}\left(s_{1}, t\right)=\bar{x}\left(s^{*}, t^{*}\right)$ with $t^{*}<t$. Then $t_{1}=t_{2}$.

Proof. 1) Any point $\bar{x}\left(s_{2}, t\right)$ is closer to $\bar{x}\left(s_{1}, 0\right)$ than it is to $\bar{x}\left(s_{2}, 0\right)$, thus a normal from $\bar{x}\left(s_{2}, t\right)$ to the initial curve is shorter than $t$. 2) By the first part, $t_{2} \leqq t_{1}$, otherwise $\bar{x}_{s_{2}}$ would have been eliminated before $t_{2}$. We now show $t_{2}=t_{1}$. Suppose not. Then $\bar{x}\left(s_{1},\left(t_{1}+t_{2}\right) / 2\right)$ is closer to $\bar{x}\left(s_{2}, 0\right)$ than to $\left(\bar{x}\left(s_{1}, 0\right)\right)$, thus there exists $s^{*}, t^{*}$ such that $\left(\bar{x}\left(s^{*}, t^{*}\right)=\bar{x}\left(s_{1},\left(t_{1}+t_{2}\right) / 2\right)\right.$ violating the hypothesis.

\section{Asymptotic States}

The proposition below states that corners decrease the variation so that the moving front approaches a circle. We shall only outline the proof; complete details may be found in [17].

Proposition 3. Let $\gamma(0)=(\alpha(s), \beta(s)), s \in[0, S]$ be a simple, closed, piecewise $C^{2}$, positively oriented initial curve. Let $\gamma(t)$ be the solution constructed from the normals by invoking the entropy condition. Then, as $t \rightarrow \infty, \gamma(t)$ approaches a circle. That is, let $\bar{\gamma}(t)$ be the front rescaled at each time so that the total length is 1 . Then, given $\varepsilon$, there exists $t_{0}$ such that for all $t>t_{0}, \bar{\gamma}(t)$ is outside a circle of radius $(1 /(2 \pi)-\varepsilon)$ and inside a circle of radius $(1 /(2 \pi)+\varepsilon)$. 
Proof. The proof consists of showing that complicated curves can be trapped between simpler curves which evolve into circles. First, a $C^{2}$ curve with everywhere positive curvature is considered. Since such a curve remains convex as it evolves, the exact solution can be used to show that the solution tends to a circle. Then, by appropriately defining what happens at corners, it can be shown that convex, piecewise $C^{2}$ curves can be trapped between explicitly constructed arbitrarily close smooth $C^{2}$ curves which by the above tend to circles. Finally, given a non-convex initial curve, its convex hull is piecewise $C^{2}$ and hence must evolve into a circle. It can then be shown that ignition curves leaving those parts of the initial curve not touching the convex hull are eliminated under the entropy condition, thus the evolution of the initial curve is eventually completely determined by its convex hull, completing the proof.

It is the development of the singularity in the curvature and the ensuing propagation of corners which stabilize and flatten the front. Linear stability analysis of this problem would show the growth of curvature, but not apply beyond the singularity when smoothness is lost. For example, the complete instability of flames which Landau predicted assumes global smoothness in time and misses this discontinuous stabilizing mechanism.

\section{Numerical Results}

\section{A. Convergence as $\varepsilon \rightarrow 0$}

Here, we show numerically that the solution for an initial cosine wave moving with $F(K)=1-\varepsilon K$ converges to the constructed weak solution as $\varepsilon \rightarrow 0^{1}$. Let $\gamma(0)=(\alpha(s)$, $\beta(s))=(s,-(\cos s+1)),-\pi \leqq s \leqq \pi$. The constructed solution for $\varepsilon=0$ which satisfies the entropy condition

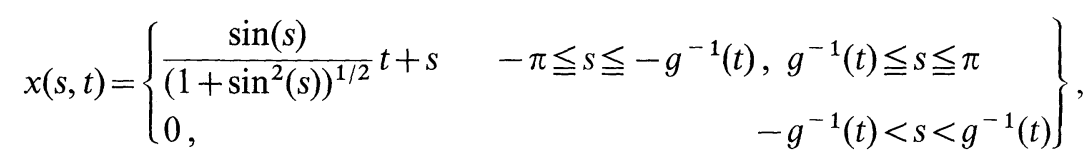

$y(s, t)$

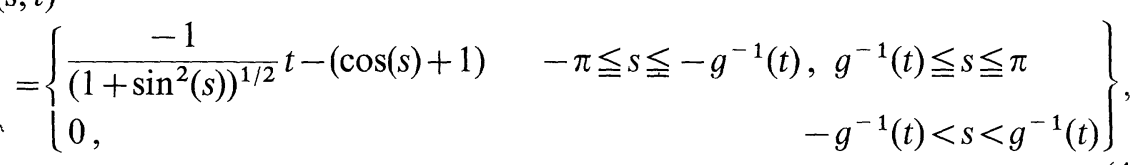

where $g(s)=|s|\left(1+\sec ^{2}(s)\right)^{1 / 2}$. A calculation shows that a corner forms at $t=1$.

To numerically solve Eq. (2.2) and (2.3) with speed $F(K)=1-\varepsilon K, \varepsilon>0$, we first tried to use the following simple numerical technique. A set of marker particles were placed along the front, and centered finite difference approximations were used for the spatial derivatives; this yielded a set of coupled ordinary differential equations for the motion of the marker particles. The time derivatives were then approximated by Heun's method. Thus, if $\left(x_{i}^{n}, y_{i}^{n}\right)$ is the position of the $\mathrm{i}^{\text {th }}$ marker particle at time $n$, then

$$
x_{i}^{n+1}=x_{i}^{n}+\frac{\Delta t}{2}\left(u_{i}+\bar{u}_{i}\right), \quad y_{i}^{n+1}=y_{i}^{n}+\frac{\Delta t}{2}\left(v_{i}+\bar{v}_{i}\right),
$$

1 The proof of a general statement under certain restrictions on the initial curve and speed function will be given elsewhere 
where

$$
\begin{gathered}
u_{i}=\left(1-\varepsilon K_{i}\right)\left(\frac{y_{i+1}-y_{i-1}}{\left.\left(x_{i+1}-x_{i-1}\right)^{2}+\left(y_{i+1}-y_{i-1}\right)^{2}\right)^{1 / 2}}\right), \\
v_{i}=\left(1-\varepsilon K_{i}\right)\left(-\frac{x_{i+1}-x_{i-1}}{\left.\left(x_{i+1}-x_{i-1}\right)^{2}+\left(y_{i+1}-y_{i-1}\right)^{2}\right)^{1 / 2}}\right), \\
K_{i}=4\left(\frac{\left(y_{i+1}-2 y_{i}+y_{i-1}\right)\left(x_{i+1}-x_{i-1}\right)-\left(x_{i+1}-2 x_{i}+x_{i-1}\right)\left(y_{i+1}-y_{i-1}\right)}{\left(\left(x_{i+1}-x_{i-1}\right)^{2}+\left(y_{i+1}-y_{i-1}\right)^{2}\right)^{3 / 2}}\right), \\
\bar{x}_{i}=x_{i}^{n}+\Delta t\left(u_{i}^{n}\right), \quad \bar{y}_{i}=y_{i}^{n}+\Delta t\left(v_{i}^{n}\right),
\end{gathered}
$$

and $\bar{u}_{i}, \bar{v}_{i}$, and $\bar{K}_{i}$ are computed using $\bar{x}_{i}$ and $\bar{y}_{i}$.

This scheme is unstable. The markers come close together where the curvature increases, and small errors in their positions can produce large errors in the curvature. Even with double precision and very small time steps, for small $\varepsilon$ it is difficult to avoid oscillations and instability.

This problem was eliminated through a regridding technique designed to keep the horizontal distance between mesh points fixed. We let $s_{1}, \ldots, s_{i}, \ldots, s_{N}$ be $N$ evenly spaced points in the interval $[-\pi, \pi], s_{1}=-\pi, s_{N}=\pi$, and let $x_{i}^{0}=\alpha\left(s_{i}\right)=s_{i}$, $y_{i}^{0}=\beta\left(s_{i}\right)$. We first use the above difference scheme to advance the points $\left(x_{i}^{n}, y_{i}^{n}\right)$ ahead one time step to produce new values $\left(x_{i}^{*}, y_{i}^{*}\right)$. Let $x_{i}^{n+1}=s_{i}$. To find $y_{i}^{n+1}$, we linearly interpolate between the points $\left(x_{i}^{*}, y_{i}^{*}\right)$ to find the intersection with the vertical line $x=s_{i}$, thus providing an updated set of marker particles $\left(x_{i}^{n+1}, y_{i}^{n+1}\right)$ such that $\left(x_{i}^{n+1}-x_{i-1}^{n+1}\right)$ is constant. We varied $N$ from 100 to 400 points and $\Delta t$ from $10^{-3}$ to $10^{-5}$ to ensure that results did not depend on grid size or time step, comparing position and total variation of the curve.

In Fig. 1, we plot $\operatorname{Var}(t)$ as a function of time for various values of $\varepsilon$, as well as $\operatorname{Var}_{\text {Ent }}(t)$ for the entropy-satisfying solution. The dashed line represents the initial value $\operatorname{Var}(0)=\pi$. Integration of (4.1-4.2) shows that $\operatorname{Var}_{\text {Ent }}(t)=\pi$ for $t<t^{*}$, where $t^{*}=|\pi / 4|\left(1+\sec ^{2}(\pi / 4)\right)^{1 / 2}$, at which time the inflection point of the curve hits the $y$ axis. As $\varepsilon \rightarrow 0$, the graphs approach that of the entropy-constructed solution,

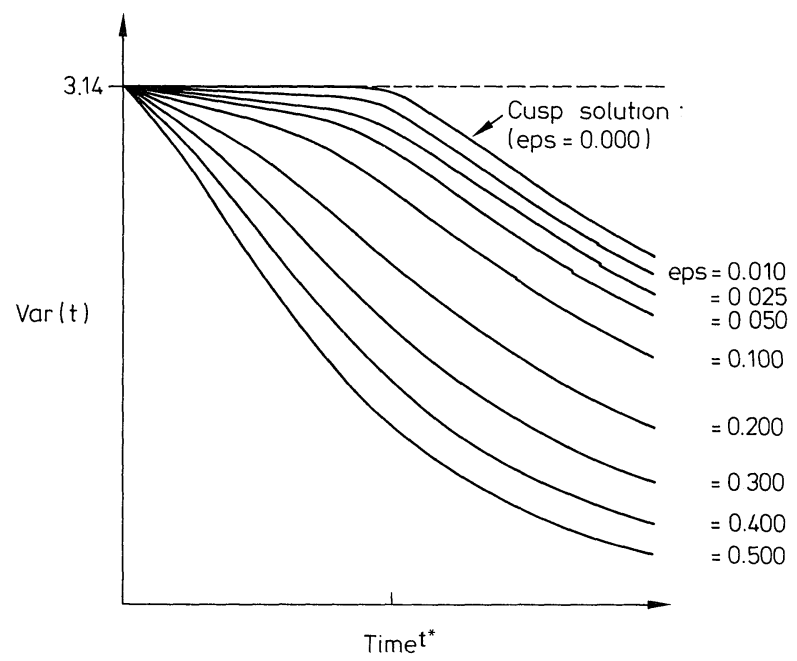

Fig. 1 


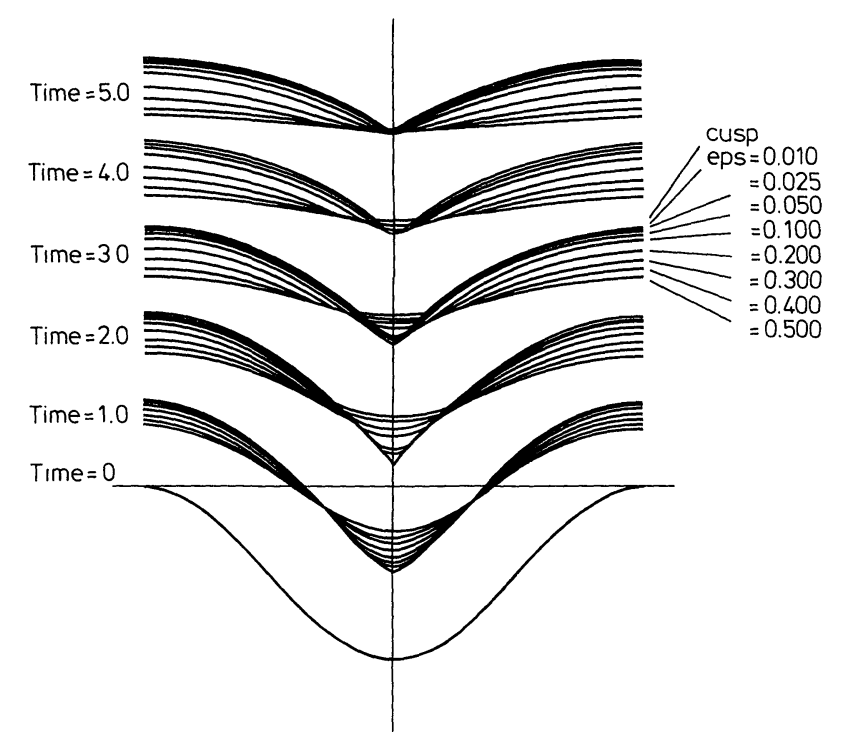

Fig. 2

Evolving curves / Varying epsilon

(labeled "cusp"), and in particular converge to the constant value $\operatorname{Var}(t)=\pi$ for $t<t^{*}$. In Fig. 2, we plot the front positions for various values of $\varepsilon$.

\section{B. Discrete Parameterization vs. Volume of Fluid Techniques}

There are problems with numerical methods based on discrete parameterizations of the front. As pointed out earlier, marker points move together when the curvature becomes very negative. Since curvature is a second derivative, small position errors cause large errors in the computed curvature. Stability for the numerical algorithm depends on the ratio of $\Delta t$ to $\Delta \alpha$, where $\Delta \alpha$ is the arclength spacing between neighboring marker points. If the marker points come close together, $\Delta \alpha$ decreases and the time step must be correspondingly decreased, making the calculation prohibitively expensive. In those regions where marker particles move far apart, a poor approximation to the curvature can result, even though the time step stability requirement may be satisfied. Reparameterization techniques which redistribute marker points depend on curvature and arclength approximations and can result in substantial smoothing. A separate problem is that if two patches burn into each other, it is difficult to decide which marker points to throw away so that those remaining form the boundary of the new combined region.

As evidence of the above, in the cosine wave calculation, drastic steps were taken to provide a solution. The front can always be written as a single-valued function over a fixed interval; the technique does not easily extend to curves that cannot be so represented. Since the solution is symmetric around $x=0$, points are spread from $-\pi$ to 0 . Symmetric boundary conditions are used at $x=0$ to insure that there was a fixed regridding line and hence a marker at the point of maximum curvature. If the calculation is instead performed over the entire interval, round-off error causes the center point to drift off the line $x=0$ and the interpolating technique used to create a new marker on that line severely smooths the curvature. 
As an alternative, numerical methods based on "volume of fluid" constructions do not rely on discrete parameterizations of the moving front and can easily handle topological issues such as merging. Such techniques can be used in conjunction with Huygens principle so that the entropy condition arises naturally. For details, see $[15,3,17]$. Here, we briefly describe one such method for the case $\varepsilon=0$, and demonstrate its use on a simple problem.

Starting with a square grid $i, j$ of uniform mesh size, a number $f_{i j}, 0 \leqq f_{i j} \leqq 1$, is assigned to each cell, corresponding to the fraction of burnt fluid within the cell. Each cell's material is moved in the direction $\mathbf{u}=(u, v)$ by executing a fractional step in each direction. An interface is drawn in the cell representing the boundary of the material, where the orientation of the interface depends on the value of $f_{i j}$ and the $f_{i j}$ 's in the cell's neighbors. This interface is then transported in the $x$ direction a distance $u \Delta t$ and the process is then repeated for the sweep in the $y$ direction, providing new $f_{i j}$.

This algorithm can be used to advance a front along its normal vector field using Huygens principle. For the moment, assume that $F(K)=1$ and consider $L$ angles, $\Theta_{l}=\left(2 \pi(l-1) / L, l=1, \ldots, L\right.$. Given any cell with volume fraction $f_{i j}^{n}$, the material in that cell is moved a unit distance in each of the $l$ directions $\left(\cos \Theta_{l}, \sin \Theta_{l}\right)$. As $L \rightarrow \infty$ and the mesh size goes to zero, this corresponds to drawing a disk of unit radius around the center of the cell. The Huygens principle construction says that the envelope formed by all such disks (that is, for all $i, j$ ) gives the front advanced a unit distance along its normal field. Thus, let $f_{i j}^{n} \Theta_{l}$ be the array of volume fractions obtained by moving the fractions in the direction $\Theta_{l}$ and let $f_{i j}^{n} \Theta_{0}=f_{i j}^{n}$. The new volume fractions approximating the front advanced one time step will be given by

$$
f_{i j}^{n+1}=\max _{0 \leqq l \leqq L} f_{i j}^{n} \Theta_{l} .
$$

Since $f_{i j}$ is often either zero or one, careful programming will limit the computing effort to the boundary. The generalization to three dimensions is straightforward.

As an example, we consider a smooth, non-convex curve that develops a corner as it moves. Let $\gamma(s)=(\alpha(s), \beta(s)), s \in[0,6 \pi]$, where $\alpha$ and $\beta$ are defined as follows:

$$
\alpha(s)=\left\{\begin{array}{cc}
-\cos (s) & 0 \leqq s \leqq \pi / 2 \\
-\cos (s) & \pi / 2 \leqq s<3 \pi / 2 \\
3 \cos (s / 3) & 3 \pi / 2 \leqq s<9 \pi / 2 \\
-\cos (s) & 9 \pi / 2 \leqq s<11 \pi / 2 \\
-\cos (s) & 11 \pi / 2 \leqq s \leqq 6 \pi
\end{array}\right\}, \quad \beta(s)=\left\{\begin{array}{cc}
\sin (s) & 0 \leqq s<\pi / 2 \\
-\sin (s)+2 & \pi / 2 \leqq s<3 \pi / 2 \\
3 \sin (s / 3) & 3 \pi / 2 \leqq s<9 \pi / 2 \\
-\sin (s)-2 & 9 \pi / 2 \leqq s<11 \pi / 2 \\
\sin (s) & 11 \pi / 2 \leqq s \leqq 6 \pi
\end{array}\right\} .
$$

Let the front propagate with speed $1 / 2$. A lengthy but straightforward calculation shows that the position of the front $(x(s, t), y(s, t))$ is given by

$$
\text { for } t \leqq 1 \text { and } \begin{aligned}
& x(s, t)=\frac{1}{2} \beta_{s} t+\alpha, \quad y(s, t)=-\frac{1}{2} \alpha_{s} t+\beta, \\
& 0 \leqq \leqq 6 \pi \\
& x(s, t)=\frac{1}{2} \beta_{s} t+\alpha, \quad y(s, t)=-\frac{1}{2} \alpha_{s} t+\beta, \\
& {\left[\cos ^{-1}\left(\frac{2}{(1+\mathrm{t})}\right)+\frac{\pi}{2}\right] \leqq s \leqq 6 \pi-\left[\cos ^{-1}\left(\frac{2}{(1+t)}\right)+\frac{\pi}{2}\right] }
\end{aligned}
$$

for $t>1$. The position of the front at various values of $t$ is shown in Fig. 3. A corner forms at $t=1$, and propagates along the positive $x$ axis. In Fig. 4, the results of the volume of fluid algorithm with 8 angles and a $60 \times 60$ mesh of cells are shown. 
Plot of equations 4.7 and 4.8
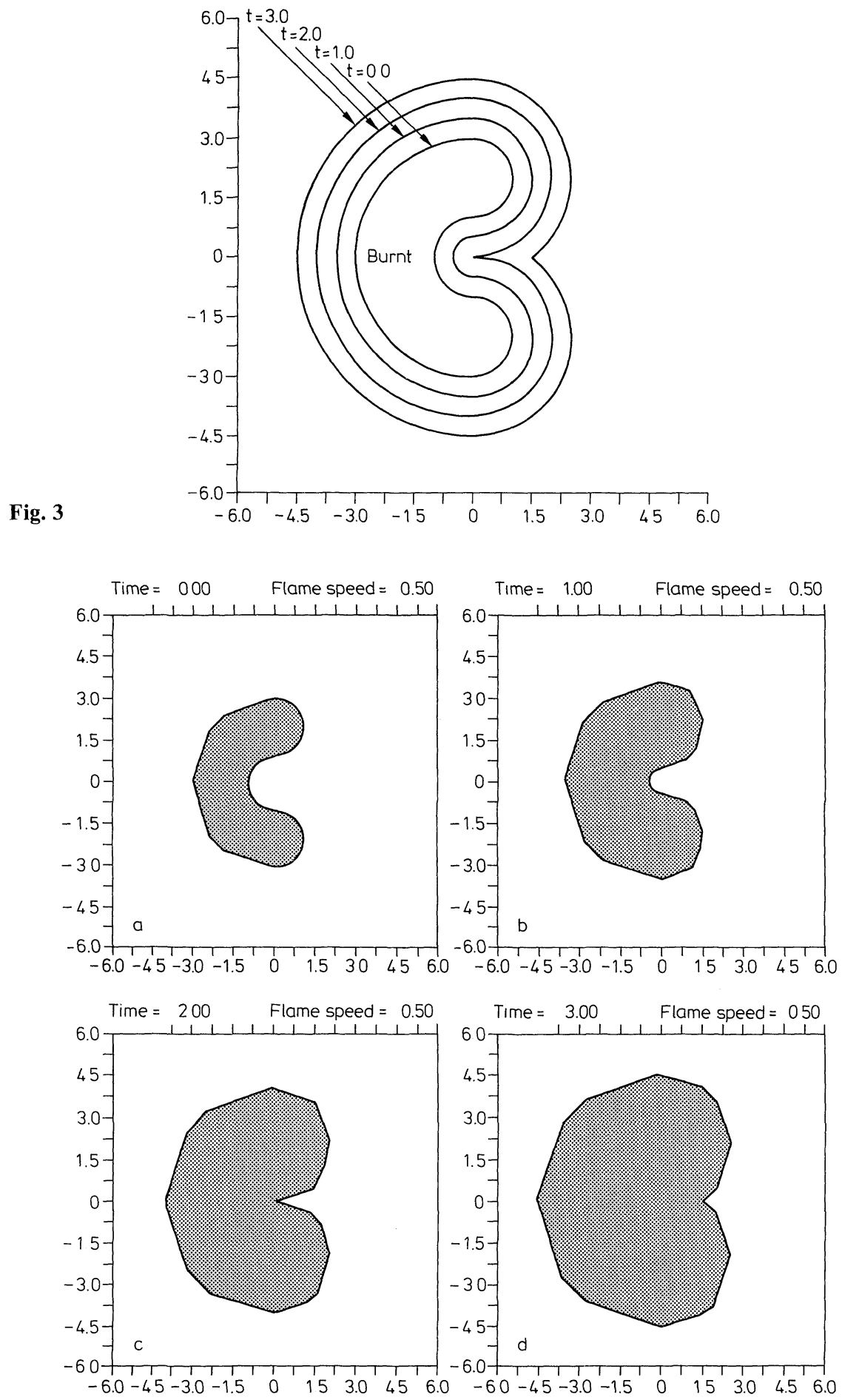

Fig. 4 
The numerical method filters out high wavelengths and smooths the solution by limiting the oscillations of the front to the order of one cell width. The method can be extended to a front moving with speed $F(K)$ by determining the curvature from the volume fractions. A method based on osculating circles has been developed [2]; there, the cell size determines the smallest possible osculating circle and hence bounds the curvature and smooths the solution.

In spite of this smoothing, such a curvature algorithm can provide a valuable tool for analyzing a moving front, since the entropy condition naturally generates weak solutions and the mesh size can be systematically refined to allow larger curvatures in a controlled way. Thus, if a mesh size $h$ is used with maximum allowable curvature $K_{h}$, the algorithm produces a weak solution with this bound; by refining the mesh size, one can investigate both possible blow up in the curvature and the nature of the solution beyond the singularity.

Acknowledgements. I would like to thank Prof. Ole Hald, Prof. Alexandre Chorin, and an anonymous reviewer for helpful suggestions and comments.

\section{References}

1. Brakke, K.A.: The motion of a surface by its mean curvature. Princeton, NJ: Princeton University Press 1978

2. Chorin, A.J.: Curvature and solidification. J. Comput. Phys. 58, 472-490 (1985)

3. Chorin, A.J.: Flame advection and propagation algorithms. J. Comput. Phys. 35, 1-11 (1980)

4. Frankel, M.L., Sivashinsky, G.I.: The effect of viscosity on hydrodynamic stability of a plane flame front. Comb. Sci. Tech. 29, 207-224 (1982)

5. Garabedian, P.R.: Partial differential equations. New York: Wiley 1961

6. Hopf, E.: The partial differential equation $u_{t}+u u_{x}=\mu u_{x x}$. Commun. Pure Appl. Math. 3, 201 (1950)

7. Huisken, G.: Flow by mean curvature of convex surfaces into spheres. Preprint (1984)

8. Landau, L.: On the theory of slow combustion.ACTA Physiocochim. URSS 19, 77-85 (1944)

9. Langer, J.S.: Instabilities and pattern formation in crystal growth. Rev. Mod. Phys. 52, 1-28 (1980)

10. Langer, J.S., Muller-Krumhaar, H.: Mode selection in a dendritelike nonlinear system. Phys. Rev. A 27, 499-514 (1983)

11. Markstein, G.H.: Experimental and theoretical studies of flame front stability. J. Aero. Sci. 18, 199-209 (1951)

12. Markstein, G.H.: Non-Steady flame propagation. New York: Pergamon Press 1964

13. Mullins, W.W., Sekerka, R.F.: J. Appl. Phys. 34, 2885 (1963)

14. Nichols, F.A., Mullins, W.W.: Trans. Met. Soc. AIME 233, 1840 (1965)

15. Noh, W., Woodward, P.: A simple line interface calculation. Proc. Fifth Intern. Conf. on Fluid Dynamics. van de Vooran, A.I., Zandberger, P.J., eds. Berlin, Heidelberg, New York: Springer 1976

16. Pamplin, B.R.: Crystal growth. New York: Pergamon Press 1975

17. Sethian, J.A.: An analysis of flame propagation. PhD. Dissertation, University of California, Berkeley, California, June 1982; CPAM Rep. 79

18. Sethian, J.A.: Turbulent combustion in open and closed vessels. J. Comput. Phys. 54, 425-456 (1984)

19. Sivashinsky, G.I.: Nonlinear analysis of hydrodynamic instability in laminar flames. I. Acta Astronaut. 4, 1177-1206 (1977)

20. Turnbull, D.: Phase changes. In: Solid state physics, Vol. 4. Seitz, F., Turnbull, D., eds. New York: Academic Press 1956

21. Zeldovich, Y.B.: Structure and stability of steady laminar flame at moderately large Reynolds number. Com. Flame 40, 225-234 (1981)

Communicated by A. Jaffe

Received November 27, 1984; in revised form March 4, 1985 
